\title{
Probe for measurements of density/conductivity in flows of conducting fluids
}

\author{
Xuejun Fan \\ Institute of Mechanics, Chinese Academy of Sciences, Beijing 100080, China \\ Garry L. Brown \\ Department of Mechanical and Aerospace Engineering, Princeton University, Princeton, New Jersey 08540
}

(Received 7 November 2005; accepted 28 February 2006; published online 3 April 2006)

\begin{abstract}
A probe utilizing the bipolar pulse method to measure the density of a conducting fluid has been developed. The probe is specially designed such that the concentration of a stream tube can be sampled continuously. The density was determined indirectly from the measurement of solution conductivity. The probe was calibrated using standard $\mathrm{NaCl}$ solutions of varying molarity and was able to rapidly determine the density of a fluid with continuously varying conductance. Measurements of the conductivity profiles, corresponding density profiles, and their fluctuation levels are demonstrated in a channel flow with an electrolyte injected from a slot in one wall.

(C) 2006 American Institute of Physics. [DOI: 10.1063/1.2189949]
\end{abstract}

\section{INTRODUCTION}

In general, for a solution of an electrolyte in water, the density of the solution is a function of electrolyte concentration, which also determines the conductivity of the solution. Thus the density of such a conducting fluid can be determined indirectly from measurements of solution conductivity.

Accurate bridge methods have frequently been used to measure solution conductivity. Typically those techniques are not appropriate for measurements over short time periods or for measurements of a continuously varying conductance because of the usual procedure that is required to adjust the impedance and balance the bridge. Instead we use a bipolar pulse technique ${ }^{1}$ developed by Johnson and Enke, which eliminates many of the classical experimental problems encountered with bridge methods and is capable of measuring very rapid changes in conductivity. This technique has subsequently been improved by Daum and Nelson for the measurement of small solution resistance (high concentration). ${ }^{2}$ This technique involves a sequential application of two successive constant current pulses of equal magnitude but of opposite sign across a conductance cell. The voltage across the cell is electronically rectified and integrated so that the area under the curve can be determined. Under some assumptions, the integrated area is directly proportional to the solution resistance for a given pulse magnitude and duration.

This article introduces the bipolar technique to measure the time resolved density/concentration over a small length scale in a conducting, turbulent fluid flow.

\section{BIPOLAR CURRENT METHOD}

The impedance of a conductance cell mainly consists of a solution resistance $R_{x}$, an electrode double layer capacitance $C_{x}$, and an impedance from Faradaic processes. The Faradaic processes are important only when the voltage across the double layer becomes sufficiently large; that is, if the applied voltage is small and its duration is sufficiently short so that the polarization, which causes the Faradaic process, is negligibly small, then a conductance cell can be simplified to a series combination of the solution resistance $R_{x}$ and the double layer capacitance $C_{x}$ in parallel to another capacitance $C_{p}$, as shown in Fig. 1. In general the parallel capacitance $C_{p}$ consists of the interelectrode capacitance and the lead/contact capacitance, and under most circumstances is much smaller than the double layer capacitance $C_{x}$, which is typically of order of $30-40 \mu \mathrm{F} / \mathrm{cm}^{2}$ in aqueous solutions.

If we assume initially that $C_{p}=0$, then when a short bipolar current pulse with a magnitude $i$ and a period of $2 \tau$ is applied to this simplified model (Fig. 1), the voltage across the cell is

$$
V_{\text {cell }}=\left\{\begin{array}{cc}
-i R_{x}\left(1+t / R_{x} C_{x}\right), & 0 \leqslant t<\tau \\
i R_{x}\left(1+(t-2 \tau) / R_{x} C_{x}\right), & \tau \leqslant t<2 \tau
\end{array}\right.
$$

and the area integrated under a rectified voltage curve is exactly equal to $2 i R_{x} \tau$. This is independent of $C_{x}$ due to the cancellation resulting from the charge and discharge of $C_{x}$. When $C_{p} \neq 0$, but $C_{p} \ll C_{x}$ and $\tau \gg R_{x} C_{p}$, the time and the current required to charge $C_{p}$ are insignificant compared to that of $C_{x}$ and the result is asymptotically the same as for $C_{p}=0$. For a small cell resistance, a large current or a longer duration is desirable to obtain a measurable signal level but the maximum values are limited by the Faradaic processes.

\section{THE DENSITY PROBE AND ITS CALIBRATION}

The bipolar pulse technique works extremely well for a uniform conductivity cell, but its application to the measurement of mean and fluctuating density/concentration profiles in a nonuniform conductivity flow field is not straightforward since the solution resistance of a conductance cell is an integral quantity over a volume of fluid. To localize the measurement, a novel probe has been designed, as sketched in Fig. 2. The probe is constructed from non-conducting tubing 


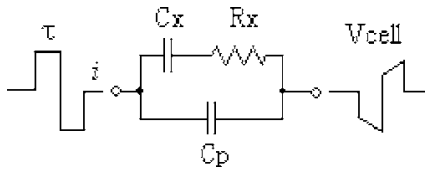

FIG. 1. Simplified equivalent circuit of a conductance cell and its response to a bipolar current pulse.

(plastic or glass) of $1.6 \mathrm{~mm}$ outside diameter, which is drawn down to approximately $1.0 \mathrm{~mm}$ with a hole at the tip of approximately $0.5 \mathrm{~mm}$ diameter. Two $0.13 \mathrm{~mm}$ platinum wires were inserted across the diameter of the tubing $12.7 \mathrm{~mm}$ apart with the first wire $3.2 \mathrm{~mm}$ downstream from the probe tip. Small diameter leads were glued to the outside of the tubing and soldered to the platinum wires. The joints and the holes were sealed with a silicone sealant (Fig. 2).

The probe tip hole diameter $D$ and the distance $L$ between the platinum wires specify the spatial resolution. For given maximum values of $i$ and $\tau$, the ratio $L / D$ will have a lower limit required to obtain a measurable cell resistance $R_{x}$. To increase the resolution in the radial direction the tip hole diameter must be reduced. Thus there is a compromise between signal strength and probe spatial and temporal resolutions.

A pressure drop is applied to the probe to establish a Poiseuille flow past the platinum wires with a velocity of the same order as the local flow velocity of the fluid in which the instantaneous concentration is to be measured. The concentration of the stream tube drawn through the probe is then determined using the bipolar pulse technique.

For the probe that has been constructed the analog control circuit and the associated control signals, which are shown in Fig. 3, are similar to those used by Daum and Nelson but simplified as a result of computer data acquisition capabilities. As can be seen from Fig. 3 the circuit consists firstly of a bipolar current source, followed by a probe control operational amplifier, a rectifier, an integrator, and a sample/hold circuit. The falling edge of the control signal $J$ starts the sampling process, and the falling edge of the signal $K$ holds the voltage and is used to trigger the computer data acquisition. The control signals $J$ and $K$ have a pulse width of $2 \tau$ and the duty cycle is programmable and determines the sampling frequency of the concentration measurements. The signals at points $A, B$, and $C$ show the bipolar current source, the probe response voltage, and the current flowing into the integrator.

To isolate electrically the probe from the conducting medium and any possible ground loop to the flow system, a battery power source was used to drive the circuit and the

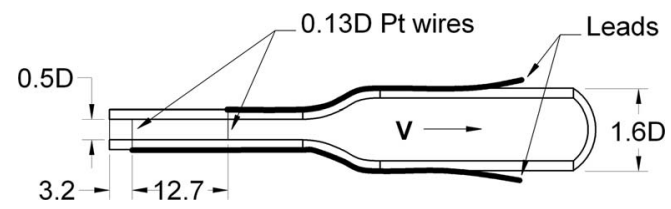

FIG. 2. Sketch of the density probe. All length dimensions are in millimeters.

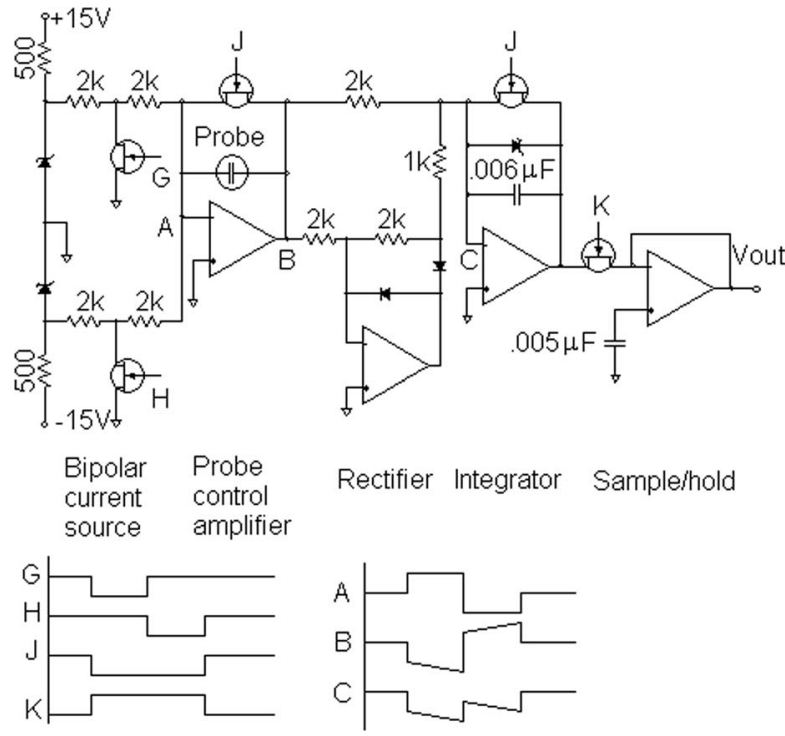

FIG. 3. Probe circuit and control signals. $A$ : Current into the amplifier; $B$ : cell response voltage; $C$ : rectified signal; and $G, H, J$, and $K$ : control voltages for the switches.

communication between the output signal and the data acquisition board in the computer was isolated using an analog optical isolator.

In the channel flow experiments for which the probe was developed, ${ }^{3}$ a pulse duration $\tau$ of $10 \mu$ s was chosen. Given the spacing of $12.7 \mathrm{~mm}$ between the platinum wires and a mean velocity of $0.3 \mathrm{~m} / \mathrm{s}$ through the probe the travel time of the flow between the electrodes is approximately $1 / 24 \mathrm{~s}$ so that measured fluctuations above $24 \mathrm{~Hz}$ cannot be readily interpreted. Fluctuations below this frequency were, however, of interest. If a higher frequency content in the density fluctuations were needed, a shorter distance between the platinum wires would be required as well as a smaller probe hole diameter to increase the resistance as stated previously. The limits are set by electrical noise and mechanical constraints on the probe geometry.

As mentioned in the Introduction, the possibility of determining the density of a conducting fluid using this system for the measurements of the conductivity arises from the dependence of both density and conductivity on the concentra-

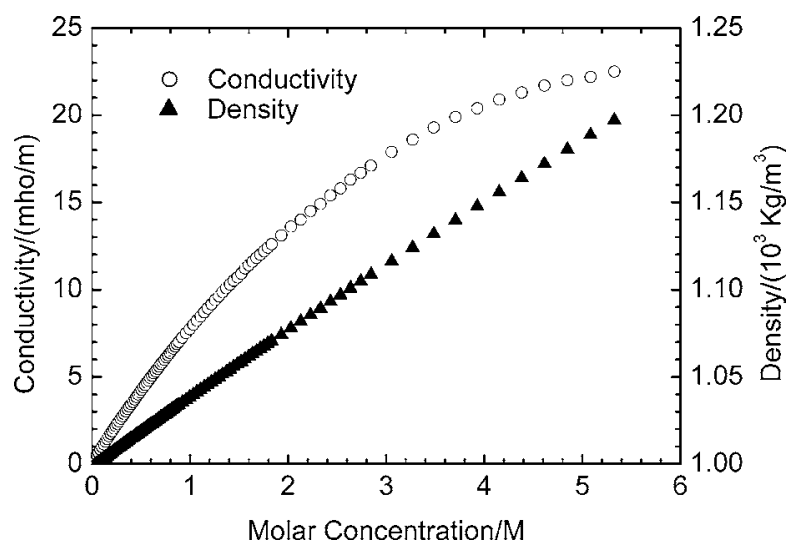

FIG. 4. Conductivity and density of $\mathrm{NaCl}$ solutions at $20{ }^{\circ} \mathrm{C}$ as a function of concentration (from Ref. 4). 


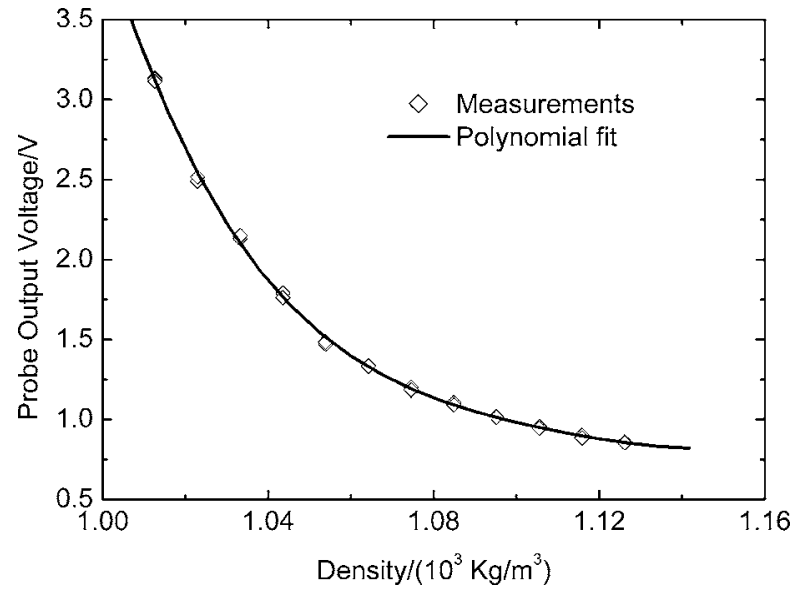

FIG. 5. Density probe calibration curve for $\mathrm{NaCl}$ solutions.

tion of the electrolyte. Figure 4, for example, shows the dependence of the density on the conductivity of aqueous $\mathrm{NaCl}$ solutions at ambient conditions $\left(20^{\circ} \mathrm{C}\right.$ and $\left.1 \mathrm{~atm}\right)$ obtained from Ref. 4. A one-to-one correspondence exists in this case between the conductivity and the density and, for example, the density $\rho$ can be calculated from the conductivity $\sigma$ using the following polynomial fit for $0<25(\mathrm{mho} / \mathrm{m})$ :

$$
\begin{aligned}
\rho= & 0.00015 \sigma^{5}-0.00690 \sigma^{4}+0.11566 \sigma^{3}-0.71901 \sigma^{2} \\
& +6.64559 \sigma+996.64739 .
\end{aligned}
$$

Solution conductivity tends towards saturation at high concentration so that a large variation in density accounts for only a small variation in conductivity at high concentrations (as is evident in Fig. 4) and thus a small variation in probe output voltage; thus there is a decreasing sensitivity in the measurement of density at high concentrations. This tendency towards saturation depends on the solute type so that prior knowledge of the solute is required to determine the sensitivity of the probe for the measurement of density or concentration.

The probe was calibrated for the measurement of the density of aqueous $\mathrm{NaCl}$ solutions using standard $\mathrm{NaCl}$ solutions of known concentration, which were prepared by dissolving a measured mass of chemically pure $\mathrm{NaCl}$ powder in $250 \mathrm{cc}$ of distilled water. The mass of $\mathrm{NaCl}$ was measured using a digital balance with a minimum scale of $0.1 \mathrm{~g}$, while the volume of water was determined using a graduated $100 \mathrm{cc}$ cylinder with a minimum scale of $1.0 \mathrm{cc}$. The uncertainty in molar concentration depends on the molarity but it was less than $5 \%$ for solution concentration higher than $0.1 M$. The solution was thoroughly stirred before being used for probe calibrations. To reduce the instantaneous fluctuations in the measurement, five successive bipolar pulses were used at a frequency of $1.0 \mathrm{kHz}$ (frequency of signal $K$, controlled by the computer) for a single measurement and an averaged output voltage of the probe was recorded. The molar concentration of the solution was converted to density using the established data in Fig. 4. The resulting calibration plot of voltage versus density is shown in Fig. 5. The response of the probe is nonlinear and the sensitivity decreases as the solution concentration/density increases. This response could be linearized or improved by adjusting the magnitude

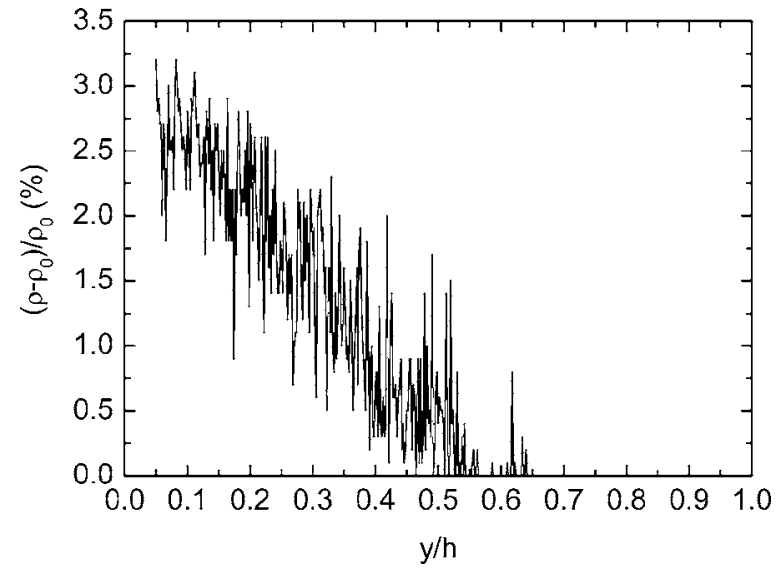

FIG. 6. Density traverse across the channel. $y$ was the vertical distance from the center of probe tip to the bottom channel wall. Channel height $h$ $=12.7 \mathrm{~mm}$.

of the current pulse for different regions of concentration. The probe provided a relatively large voltage signal and gave highly repeatable results. The overall errors in density calibrations were approximately $5 \%$ of the density difference between the measured value and one with a molarity of zero for molar concentration higher than $0.1 M$ (note from Fig. 4 the nearly linear relationship between density and molar concentration).

\section{MEASUREMENTS AND DISCUSSION}

Measurements of density distributions with this probe were carried out in a two-dimensional turbulent channel flow in which a dense fluid was injected eight channel heights upstream from a slot in the lower wall of the horizontal channel. The channel height was $12.7 \mathrm{~mm}$ and the mean flow velocity was approximately $0.6 \mathrm{~m} / \mathrm{s}$. Figure 6 shows an example of the measurements made during a traverse of the probe across the channel for the case when a $4.0 \mathrm{M} \mathrm{NaCl}$ solution was injected from the upstream slot. The output voltages have been converted to density based on the calibration shown in Fig. 5. The results are actually a series of discrete measurements, one from each sampling point. The probe was moved using a stepper motor in steps of $0.025 \mathrm{~mm}$ and at a rate of $100 \mathrm{~Hz}$ so that the traverse of $10 \mathrm{~mm}$ provided 400 discrete measurements. It can be seen that the local density fluctuations only occur below $y / h$ $=0.7$. One can estimate an average spacing for the measured density fluctuations of approximately $0.01 \mathrm{~h}$, which corresponds to five steps of the traversing probe or $0.05 \mathrm{~s}$ in time. Thus a characteristic frequency from this measurement was of the order of $20 \mathrm{~Hz}$. Turbulent fluctuations at higher frequencies have been filtered by the probe. This frequency can be compared with a characteristic frequency of $47 \mathrm{~Hz}$ obtained from the mean flow velocity and the channel height. The mean density profile can be obtained from an ensemble average of polynomial fits to such data. 
It should be noted that the $12.7 \mathrm{~mm}$ spacing between the platinum wires was chosen in this study because a higher frequency response was not needed and a large signal was an advantage. The output voltages for this spacing were on the order of $1 \mathrm{~V}$ (Fig. 5). There should be no difficulty in reducing $L$ and measuring much smaller voltages and then the frequency response of the probe could be increased to the order of $1 \mathrm{kHz}$ or higher. A smaller probe hole diameter would be required to improve the spatial resolution.

\section{ACKNOWLEDGMENT}

This work was partially funded by the U.S. Navy Naval Undersea Warfare Center (NUWC) and their interest and support are much appreciated.

${ }^{1}$ D. E. Johnson and C. G. Enke, Anal. Chem. 42, 329 (1970).

${ }^{2}$ P. H. Daum and D. F. Nelson, Anal. Chem. 45, 463 (1973).

${ }^{3}$ X. Fan, Ph.D. thesis, Princeton University, 2000.

${ }^{4}$ Handbook of Chemistry and Physics, 57th ed. edited by R. C. Weast (CRC, Cleveland, OH, 1977), pp. D252-D253. 